\title{
Compact accurate scalable model for millimeter wave InP CPW with under-bridge
}

\author{
Gang Liu ${ }^{1,2}$, Hiroshi Nakano ${ }^{3}$, and Kazuhiko Honjo ${ }^{1 \mathrm{a})}$ \\ ${ }^{1}$ Department of Information and Communication Engineering, University of \\ Electro-Communications, Tokyo, 182-8585 Japan. \\ ${ }^{2}$ School of Electronic Engineering, University of Electronic Science and \\ Technology of China (UESTC), Chengdu, 610054, China. \\ ${ }^{3}$ AMMSYS Inc., Incubation Center, Tokyo Institute of Technology, Tokyo, \\ 152-8552 Japan. \\ a) honjo@ice.uec.ac.jp
}

Abstract: A compact accurate scalable model for CPW on $\mathrm{InP}$ with a single under-bridge is successfully established and testified by both measurements and EM simulations up to $50 \mathrm{GHz}$. Then it helps realize a novel matching network design consisting of only two underbridges beneath CPW, with the functions of both even mode impedance matching and odd mode suppression.

Keywords: CPW, millimeter wave, InP, MMIC, mode suppression Classification: Microwave and millimeter wave devices, circuits, and systems

\section{References}

[1] N. I. Dib et al., "Analysis of Shielded CPW Discontinuities with AirBridges," Microwave Symposium Digest, IEEE MTT-S International, pp. 469-473, 1991.

[2] J. Hu and L. Sun, "EC-ANN models for CPW discontinuities," IEE Proc. Microw. Antennas Propag., vol. 152, no. 5, pp. 292-297, Oct. 2005.

[3] A. A. Omar et al., "Effects of air-bridges and mitering on coplanar waveguide 90 degrees bends: theory and experiment," Microwave Symposium Digest, IEEE MTT-S International, pp. 823-827, 1993.

[4] P. Sewell and T. Rozzi, "Characterization of air-bridges in MM-wave coplanar waveguide using the complete mode spectrum of CPW," vol. 42, no. 11, pp. 2078-2087, Nov. 1994.

[5] W. R. Deal et al., "Demonstration of a 270-GHz MMIC Amplifier Using 35-nm InP HEMT Technology," IEEE Microwave Wireless Comp. Lett., vol. 17, no. 5, pp. 391-393, May 2007.

[6] S. Gevorgian, L. J. Peter Linner, et al., "CAD Models for Shielded Multilayered CPW," IEEE Trans. Microwave Theory Tech., vol. 43, no. 4, pp. 772-779, April 1995.

[7] R.-B. Wu et al., "Inductance and Resistance Computations for ThreeDimensional Multiconductor Interconnection," IEEE Trans. Microwave Theory Tech., vol. 43, no. 4, pp. 263-271, April 1995. 
[8] E. Hammerstad et al., "Accurate Models for Microstrip Computer-Aided Design," Microwave Symposium Digest, MTT-S International, vol. 80, no. 1, pp. 407-409, May 1980.

\section{Introduction}

The InP-based HEMT has demonstrated high-frequency characteristics superior to other transistor in millimeter wave and even higher band. And coplanar waveguide (CPW) is also a preferred medium for MMIC applications. In CPW circuits, air-bridges are commonly used to suppress parasitic odd mode signals. However, they introduce parasitic parameters that people proposed various analytical models and numerical methods to evaluate $[1,2,3,4]$ and hence to minimize in the past.

If appropriately made use of these 'parasitic parameters', there will be a win-win solution: we can boldly increase the bridge size for superior odd mode suppression, meanwhile get a usable circuit elements. A model of CPW with bridge that is not only accurate and scalable but also compact hence fast enough for the practical schematic simulation and optimization will be essential for solution.

Such a model is proposed in this paper and helps to realize a novel impedance matching method. Instead of air-bridge, under-bridge is used due to its better mechanical strength as well as potentially higher accuracy (rather than the square structure simplified in many model analysis, airbridge is actually arc shaped [5]), and its compact accurate scalable model is established and verified by measurements and EM simulations in Chapter 2. It is straight-forward analytical formula based, and with modeling idea different from the 'hybrid technique' in [1], the structure of bridge under CPW is considered as an integrated entity. The model is then used as basic circuit element to compose a matching network in Chapter 3, realizing both even mode impedance matching and superior odd mode suppression, with a satisfactory MAG.

\section{MODEL OF CPW WITH SINGLE BRIDGE}

\subsection{Circuit Constitution}

As shown in Fig. 1, the fabricated InP CPW has a bridge which goes between the top layer of $\mathrm{BCB}$ and lower layer of InP, to connect two side grounds. Its corresponding Equivalent Circuit consists three parts with central symmetry: CPW part, mode conversion (MC) part and MicroStrip (MS) part, which will be discussed in detailed respectively.

\subsection{CPW part}

The Equivalent Circuit of CPW part is a conventional LC T-network, with series loss resistors. The effective relative permittivity $\varepsilon_{\text {eff }}$ and characteristic impedance $\mathrm{Z}_{0}$ of $\mathrm{CPW}$ is derived from analytical expressions for back 
grounded CPW with two layers substrates, based on quasi-static TEM conformal mapping technique [6], and then converted into LC values of T network, according to the formulas

$$
\begin{gathered}
L=\mathrm{Z}_{0} \times \mathrm{Lth} \times \mu_{\mathrm{o}} \times \sqrt{\varepsilon_{\mathrm{eff}}} / \eta_{\mathrm{o}} \\
\mathrm{C}=1 / \mathrm{Z}_{0} \times \operatorname{Lth} \times \varepsilon_{\mathrm{o}} \times \sqrt{\varepsilon_{\mathrm{eff}}} \times \eta_{\mathrm{o}}
\end{gathered}
$$

where $\mathrm{L}_{\mathrm{th}}$ is the length of CPW, and ideally $\mathrm{C}$ is the sum of the capacitance between signal line to side ground and to back ground. However, due to the absence of over through via hole in the fabricated structure, back metal plate is not a literally as a perfect ground as side ground (test probe station platform is not perfectly RF grounded either), introducing additional capacitances coupling back metal and side grounds, which consequently compensate the total value of $\mathrm{C}$, by a factor ' $\mathrm{a}$ ', which is decided empirically by the measurement results of fabricated CPW without bridges, thus

$$
\mathrm{C}=\mathrm{a} / \mathrm{Z}_{0} \times \mathrm{Lth} \times \varepsilon_{\mathrm{o}} \times \sqrt{\varepsilon_{\mathrm{eff}}} \times \eta_{\mathrm{o}}
$$

$$
\mathrm{a}=0.77
$$

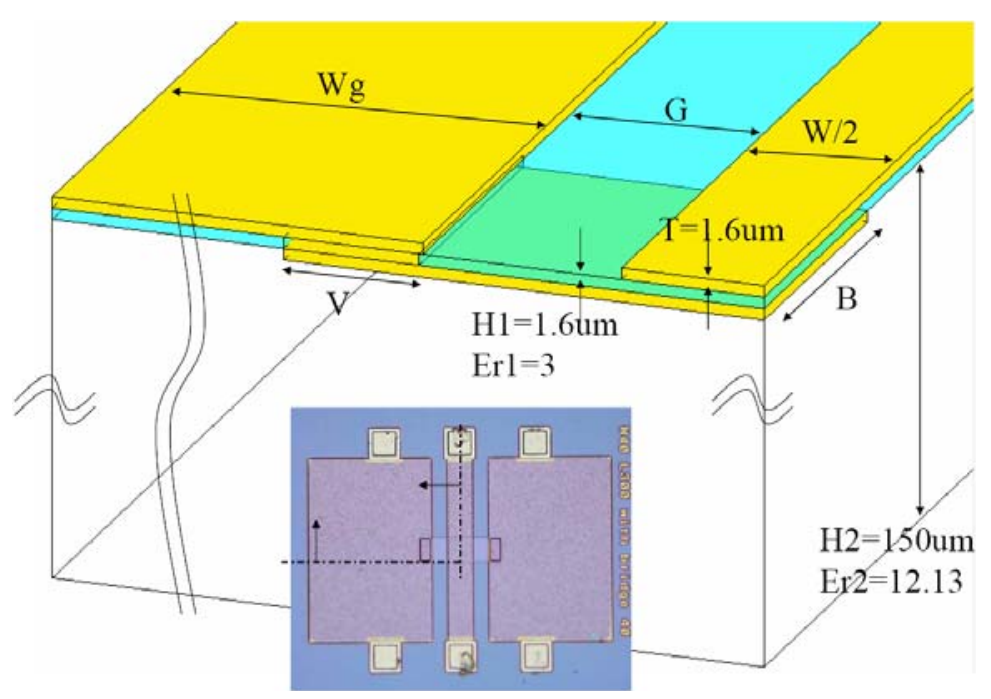

Fig. 1. Photo and its cross section view of CPW with under-bridge. Length unit: um.

In the calculation of metal loss series resistance, skin effect is taken into account. Considering that traditional skin depth formula $\delta=1 / \sqrt{\pi \mu \sigma f}$ can easily increase beyond the real metal thickness $\mathrm{T}$, until infinite, as frequency f decreases towards DC; 'effective area' [7] is used:

$$
\mathrm{A}_{\mathrm{eff}}=2(\mathrm{~W}+\mathrm{T}) \delta \frac{1-\frac{1-\alpha}{1+\alpha} \mathrm{e}^{-\gamma \varepsilon}-\frac{2 \alpha}{1+\alpha} \frac{1-\mathrm{e}^{-\gamma \varepsilon}}{\gamma \varepsilon} \mid}{\left|1-\frac{1-\alpha}{1+\alpha} \mathrm{e}^{-2 \varepsilon}-\frac{\alpha}{1+\alpha} \frac{1-\mathrm{e}^{-2 \varepsilon}}{\varepsilon}\right|}
$$


where $\gamma=1+\mathrm{j}, \alpha=\mathrm{T} / \mathrm{W}, \varepsilon=\mathrm{T} / 2 \delta$. And signal line's series loss is calculated by

$$
\mathrm{R}_{\mathrm{cpw}}=\frac{\mathrm{Lth}}{\sigma \times \mathrm{A}_{\mathrm{eff}}}
$$

When $\mathrm{f}$ decreases, $\mathrm{A}_{\text {eff }}$ will increase towards the maximum limit, the practical signal line cross section area.

The equivalent circuit for CPW part is verified by measurement results.

\subsection{MS part}

Equivalent Circuits of MS part is shown in Fig. 2 (a). Analyzing the bridge structure in Fig. 1, we can see that since $G$ is much larger than $\mathrm{H}_{1}$, it's reasonable to assume the dominant mode to be quasi-microstrip mode (not an ideal microstrip mode either), rather than CPW mode; and such assumption enable us to borrow microstip line analysis method to analyze this structure, considering signal line, top dielectric layer $\left(\varepsilon_{\mathrm{r} 1}=3\right)$ and under-bridge as signal line, MS substrate and back ground in MS mode respectively.

With equations (1) (2), $\mathrm{L}_{\mathrm{ms}}$ and $\mathrm{C}_{\mathrm{ms}}$ can be converted from MS effective relative permittivity $\varepsilon_{\text {eff }}$ and characteristic impedance $Z_{0}$, which could be obtained by Hammerstad's model [8]. The fundamental factor is defined as $\mathrm{u}=\mathrm{W} / \mathrm{H}_{1}$, and it was declared that the accuracy of this MS model is better than $0.003 \%$ for $u \leq 1000$. Though the ratio ' $u$ ' still falls well into the valid range in our case, the absolute values of configuration $(\mathrm{W}=40,80 \mathrm{um}$, $\mathrm{H}_{1}=1.6 \mathrm{um}$ ) make it hard to form the ideal MS mode, thus modification to Hammerstad's model becomes necessary to fit the case. According to the measurements and EM simulations, $u$ is empirically redefined as

$$
\begin{gathered}
\mathrm{u}=\mathrm{b}(\mathrm{B}, \mathrm{W}) \times \frac{\mathrm{W}}{\mathrm{H}_{1}} \\
\mathrm{~b}(\mathrm{~B}, \mathrm{~W})=2+0.8 / \exp \left(\frac{\mathrm{B} \times 10^{6}-40}{19}+\frac{\mathrm{W} \times 10^{6}-40}{180}\right)
\end{gathered}
$$

And in addition to LC values of conventional T network, self inductances [6] of bridge and via holes that connect side grounds and bridge, namely $\mathrm{L}_{\mathrm{msb}}$ and $\mathrm{L}_{\mathrm{msv}}$ are also added, as a current route from MS ground to CPW side ground. And concerning the existence of side grounds, whose effect is not included in the conventional MS model, $\mathrm{C}_{\text {edge }}$ is used to calculate the edge coupling between signal line and side grounds, though insignificant. Metal loss series resistance is calculated in the same way as in CPW part.

\subsection{MC part}

The Mode Conversion (MC) part is an inductance that connects CPW part and MS part in series, describing the current distribution change due to mode conversion between CPW and MS. And the inductance value is empirically defined as:

$$
\mathrm{L}_{\mathrm{mc}}(\mathrm{B}, \mathrm{W})=19 \times 10^{-12} \exp \left[-\left(\frac{\mathrm{B} \times 10^{6}-20}{80}\right)^{\frac{\mathrm{w} \times 10^{6}}{20}-1}\right]
$$


Till now, the Model for CPW with under-bridge is established, with input parameters of only physical configurations.

\subsection{Experiment Verifications}

In order to testify our model, CPW with under-bridge structures with various configurations are fabricated. S11 results of measurements, EM Simulations and model are compared in Fig. 2. We can see that at least in the range of $40 \mathrm{um} \leq \mathrm{W} \leq 80 \mathrm{um}$, and $20 \mathrm{um} \leq \mathrm{B} \leq 200 \mathrm{um}$, up to $50 \mathrm{GHz}$, our model has a perfect accordance with both measurements $(B=20$ and $40 \mathrm{um})$ and $\mathrm{EM}$ simulations (EM simulation models firstly verified by experiments help to expand the range of $\mathrm{B}$ to larger values that are practical as circuit elements in chapter 3 ).

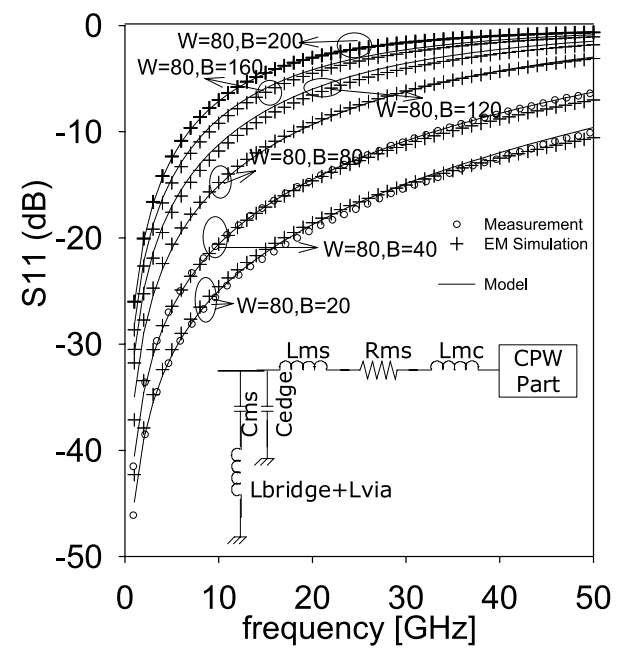

(a)

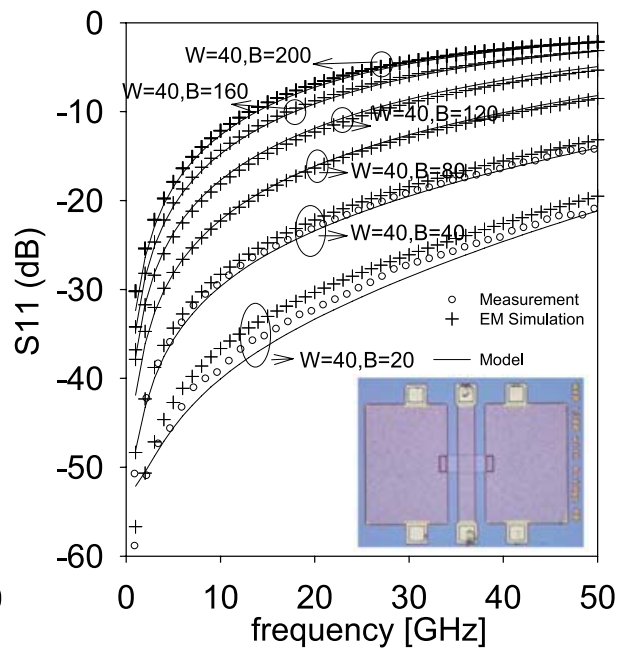

(b)

Fig. 2. CPW with under-bridge's S11 results of Measurements, EM Simulations and Model, where B ranges from $20 \mathrm{um}$ to $200 \mathrm{um}$ (measurements have only $\mathrm{B}=20$ and $40 \mathrm{um}$ ); $\mathrm{W}=80 \mathrm{um}$ in (a) and $40 \mathrm{um}$ in (b). Model with mirror symmetry is also shown in (a), and one of experiement patent's photo in (b).

\section{Matching Network}

\subsection{Matching network model}

As a novel matching approach, we propose to use under-bridges to compose a matching network. Due to the limitation of shunt effective capacitance provided by a single bridge, two different sized under-bridges are connected in series as a matching network, with a practical impedance matching capability. The corresponding equivalent circuit is established by composing two CPW with single bridge equivalent circuits in series, sharing a common CPW part. 


\subsection{Impedance matching}

Matching network's layout and impedance matching target are shown in Fig. 3 (a). With the help of model we get in Chapter 3.1, schematic simulation is executed, where bridges widths $\mathrm{B}_{1}, \mathrm{~B}_{2}$ and three part CPW lengths $\mathrm{Lth}_{1}$, $\mathrm{Lth}_{2}, \mathrm{Lth}_{3}$ are optimized (all the rest are settle in advance, i.e. $\mathrm{W}=40 \mathrm{um}$, $\mathrm{G}=28 \mathrm{um})$, towards the goals of noise optimum matching $\left(\mathrm{Z}_{\mathrm{opt}}=31.1+\mathrm{j} 37.6\right.$ at $44 \mathrm{GHz}$ ) with minimum total circuit size. Such process takes very short time on a common used desktop PC.

In order to verify the Optimized configuration yielded by model, an EM simulation model is built accordingly $\left(\mathrm{Lth}_{1}=50, \mathrm{Lth}_{2}=298\right.$ and $\mathrm{Lth}_{3}=122.2$; $\mathrm{B}_{1}=145.6, \mathrm{~B}_{2}=60.05 \mathrm{um}$ ) and simulated. Fig. 3 (a) shows that the $\mathrm{S} 22$ result of model precisely reaches the impedance target, and has good accordance with EM simulation.

\subsection{Odd mode suppression}

As the original and main reason of bridge's existence, odd mode suppression effect is an essential concern. As shown in Fig. 3 (b), according to odd mode S21 result yielded by EM simulations, our novel matching network demonstrates $10 \mathrm{~dB}$ better odd mode suppression than CPW with a single under-bridge, whose $\mathrm{W}=40, \mathrm{~B}=20$ and $40 \mathrm{um}$ respectively.

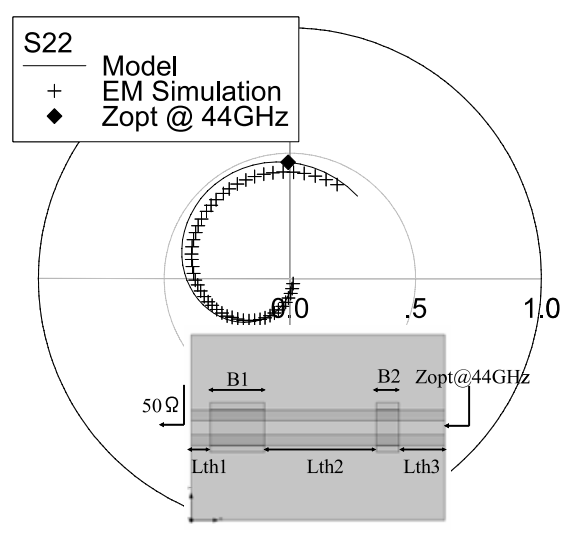

(a)

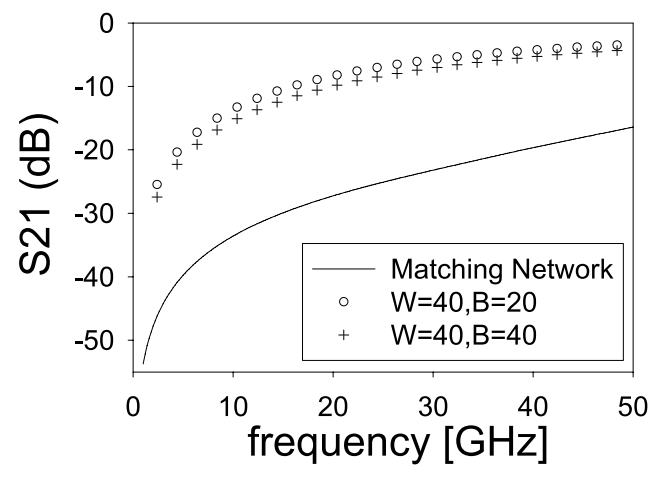

(b)

Fig. 3. (a) Matching network's S22 results of Model and EM Simulation in Smith chart; as well as matching network layout and optimization variables in lower part. (b) Odd mode suppression simulation results of $\mathrm{CPW}$ with single bridge of $\mathrm{B}=20$, $\mathrm{B}=40(\mathrm{um})$ and matching network $(\mathrm{W}=40$ for all the cases).

\subsection{Even mode MAG}

In order to exam the even mode transmission loss of this matching network, Maximum available gain (MAG) analysis is carried out, based on the S parameters obtained from EM simulation. The matching network demonstrates 
no more than $-0.2 \mathrm{~dB}$ MAG up to $50 \mathrm{GHz}$.

\section{Conclusion}

The compact accurate scalable model for millimeter wave InP CPW with under-bridge introduced in this paper makes it possible and convenient to both realize impedance matching and improve odd mode suppression at the same time, with very small loss.

\section{Acknowledgments}

The authors would like to thank Dr. N. Kawaguchi of NAOJ and Mr. T. Ohno of Japan Communication Equipment Co, Ltd. for discussions. 